\title{
Two- to three-dimensional crossover in a dense electron liquid in silicon
}

\author{
Guy Matmon,,${ }^{1 *}$ Eran Ginossar, ${ }^{2}$ Byron J. Villis, ${ }^{1}$ Alex Kölker, ${ }^{1}$ Tingbin Lim,,${ }^{1}$ Hari Solanki, ${ }^{1}$ Steven R. Schofield, ${ }^{1}$ \\ Neil J. Curson, ${ }^{1}$ Juerong Li, ${ }^{2}$ Ben N. Murdin, ${ }^{2}$ Andrew J. Fisher, ${ }^{1}$ and Gabriel Aeppli ${ }^{3}$ \\ ${ }^{1}$ London Centre for Nanotechnology, University College London, 17-19 Gordon Street, London WC1H 0AH, United Kingdom \\ ${ }^{2}$ Advanced Technology Institute, University of Surrey, Guildford GU2 7XH, United Kingdom \\ ${ }^{3}$ Institute of Physics, Ecole Polytechnique Fédérale de Lausanne (EPFL), 1015 Lausanne, Department of Physics, ETH Zürich, 8093 Zürich, \\ and Paul Scherrer Institute, 5232 Villigen, Switzerland
}

(Received 14 February 2018; published 23 April 2018)

\begin{abstract}
Doping of silicon via phosphine exposures alternating with molecular beam epitaxy overgrowth is a path to Si:P substrates for conventional microelectronics and quantum information technologies. The technique also provides a well-controlled material for systematic studies of two-dimensional lattices with a half-filled band. We show here that for a dense $\left(n_{s}=2.8 \times 10^{14} \mathrm{~cm}^{-2}\right)$ disordered two-dimensional array of $\mathrm{P}$ atoms, the full field magnitude and angle-dependent magnetotransport is remarkably well described by classic weak localization theory with no corrections due to interaction. The two- to three-dimensional crossover seen upon warming can also be interpreted using scaling concepts developed for anistropic three-dimensional materials, which work remarkably except when the applied fields are nearly parallel to the conducting planes.
\end{abstract}

DOI: 10.1103/PhysRevB.97.155306

\section{INTRODUCTION}

The possibility of using single phosphorus (P) atoms in silicon as qubit hosts has driven the development of a fabrication technology for deterministic placement of the dopants, which is very different from ordinary ion implantation/annealing paradigms. Hydrogen-passivated surfaces of Si(100) wafers are edited using a scanning tunneling microscope tip which removes protons, leaving behind exposed regions of silicon which can selectively bind phosphine $\left(\mathrm{PH}_{3}\right)$ molecules to incorporate $\mathrm{P}$ in the semiconductor. Subsequent annealing and silicon deposition then yields encapsulated twodimensional $\mathrm{P}$ structures; iteration of all steps in this process can provide three-dimensional (3D) devices [1,2].

Important components of any integrated electronic device technology are highly conducting sheets which can serve as current vias or capacitance/shielding plates, and this is accomplished by simply exposing large unpassivated areas of Si to phosphine. The result is very heavily doped twodimensional silicon, with typically $1 / 180 \mathrm{P}$ atoms/Si atom (taking into account the actual layer thickness, as referred to later). For comparison, the solubility limit of $\mathrm{P}$ in bulk CZ-grown $\mathrm{Si}$ is 1/450-1/250 impurities/Si atoms [3,4], and the highest densities for activated subsurface ion implanted layers correspond to $1 / 80 \mathrm{P} / \mathrm{Si}$ [5]. Furthermore, for the two-dimensional electron gases produced in $\mathrm{Si}$ metal-oxidesemiconductor field-effect transistors (MOSFETs), the carrier densities are typically $10^{12}-10^{13} \mathrm{~cm}^{-2}$, corresponding to 1/5000-1/50000 electrons/Si, where we assume an inversion layer thickness of $10 \mathrm{~nm}$ [6]. Table I shows that the $\delta$-layer technique produces the densest two-dimensional (2D) systems while retaining the coherence characteristics.

\footnotetext{
*g.matmon@ucl.ac.uk
}

Beyond their potential for conventional and quantum electronics, the $\mathrm{P} \delta$-layers are of fundamental physical interest because they realize a disordered 2D Hubbard model where the sites are provided by the $\mathrm{P}$ dopants. This unprecedented high density should allow us access to what should be the simple (disordered) Fermi liquid regime for large $t / U$, where $t$ is the hopping integral and $U$ is the on-site Coulomb interaction, where classical weak-localization (WL) theory should become exact.

We have consequently set out to test this hypothesis, taking advantage not only of high quality $\delta$-layers of phosphorus in silicon, but also of a modern vector magnet, which allows fields to be applied in arbitrary directions under software control. The density of dopants in the conducting slab in our sample is $2.8 \cdot 10^{14} \mathrm{~cm}^{-2}$, which corresponds to a typical interimpurity spacing of $1.5 \mathrm{~nm}$, approximately the Bohr radius for $\mathrm{P}$ in Si. Theoretical estimates [10,11] of the Hubbard model parameters for $\mathrm{Si}: \mathrm{P}$ show for interimpurity spacing of $0.75 \mathrm{~nm}$, the unscreened on-site energy $U \approx 40 \mathrm{meV}$ would be similar to the tunneling rate $t$. Additionally, some reduction of $U$ is expected due to screening and hence it is likely that the experimental regime that we study here is well described by the metallic regime of the Hubbard model [12]. On this backdrop, the focus of this paper is a full-temperature, magnitude-, and angle-dependent magnetoconductance (MC) set of experiments showing unprecedentedly rigorous demonstration of the validity of WL theory in two dimensions, including the remarkably simple formula where the in-plane field component, due to the thickness of the finite $\delta$-layer, simply increases the inelastic scattering rate to be inserted into the Hikami-Larkin-Nagaoka WL formula [13] form (which is valid in the limit of zero thickness and depends only on the perpendicular component):

$$
\frac{1}{\tau_{\varphi}} \rightarrow \frac{1}{\tau_{\text {eff }}}=\frac{1}{\tau_{\varphi}}+\frac{1}{\tau_{B}},
$$


TABLE I. Typical growth and transport parameters for different 2D systems. $L$ and $L_{\varphi}$ are the elastic and inelastic scattering lengths, respectively. $n_{s}$ and $n$ are sheet and bulk electron densities, respectively, and $d$ is the layer thickness.

\begin{tabular}{lccllc}
\hline \hline 2D system & $\begin{array}{c}L \\
{[\mathrm{~nm}]}\end{array}$ & $\begin{array}{c}L_{\varphi} \\
{[\mathrm{nm}]}\end{array}$ & $\begin{array}{c}n_{s} \times 10^{14} \\
{\left[\mathrm{~cm}^{-2}\right]}\end{array}$ & $\begin{array}{c}n \times 10^{21} \\
{\left[\mathrm{~cm}^{-3}\right]}\end{array}$ & $\begin{array}{c}d \\
{[\mathrm{~nm}]}\end{array}$ \\
\hline Ge:P $\delta$-layer [2] & 5 & 184 & 0.63 & 0.45 & 1.49 \\
Current work & 15 & 320 & 2.8 & 0.28 & 9.44 \\
Si MOSFET [7] & 48 & 252 & 0.07 & 0.007 & 10 \\
QW 2DHG [8] & 26 & 61 & $4.5 \cdot 10^{-4}$ & $2.25 \cdot 10^{-5}$ & 20 \\
Graphene [9] & 50 & 900 & 0.005 & $\sim 0.005$ & $\sim 1$ \\
\hline \hline
\end{tabular}

where $\tau_{\varphi}$ is the inelastic scattering time and $\tau_{B}$ is the additional phase-breaking time due to $B_{\|}$. Mensz and Wheeler [14] originally demonstrated the applicability of Eq. (1) for silicon MOSFETs in tilted fields, and Mathur and Baranger [15] later added substantial theoretical justification.

The form of the Hikami-Larkin-Nagaoka formula with $\tau_{\text {eff }}$, where $B_{\|}$and $B_{\perp}$ are not interchangeable, is qualitatively different from anisotropic 3D metals where the field components are interchangeable. We have exploited the vector magnet to explore the growth of this difference on cooling, and have discovered another remarkably simple form which characterizes all data except those with $\boldsymbol{B}$ nearly parallel to the planes:

$$
\Delta \sigma^{p}=\Delta \sigma_{\|}^{p}+\Delta \sigma_{\perp}^{p} .
$$

We associate $2 / p$ with a generalized anisotropic power-law approximation of Hikami's WL expression. In the case of isotropic, 3D disordered metals, the power law $p$ is confined to vary between 1 and 4 [16], i.e., values larger than 4 are not allowed. In the limit where the dephasing length is short compared to the magnetic length $\sqrt{\Phi_{0} / B}$ (where $\Phi_{0}$ is the magnetic flux quantum), the power-mean $p$ approaches unity, meaning that conductances affected by scattering parallel and perpendicular to the plane simply add as for parallel resistor shunts. In the opposite limit $p$ becomes very large, meaning that whichever conductance is larger, namely, that due either to in-plane or out-of-plane scattering, dominates entirely.

We also find a collapse of the tilted-field MC onto the perpendicular-field MC by using an angle-dependent anisotropic scaling. We associate the scaling parameter $\xi_{0}$ with the effective anisotropy of the system. Together, we show that $p$ and $\xi_{0}$ map the WL correction at arbitrary field angles and magnitudes for a given system.

\section{DEVICE FABRICATION AND MEASUREMENT}

Our test device was grown by saturation-dosing of $\mathrm{PH}_{3}$ on a $\mathrm{Si}(100)$ flat surface, annealing for $2 \mathrm{~min}$ at $350{ }^{\circ} \mathrm{C}$, then overgrowing $15 \mathrm{~nm}$ of silicon at $250{ }^{\circ} \mathrm{C}$ without the use of a locking layer $[17,18]$. This is similar to the work by Goh et al. [19]. The finished $\delta$-layer was processed into Hall bars, with mesa dimensions $94 \times 20 \mu \mathrm{m}^{2}$. Scanning tunneling microscopy measurements yielded a nominal sheet density of $2 \times 10^{14} \mathrm{~cm}^{-2}$, and the measured Hall sheet density was $2.8 \times 10^{14} \mathrm{~cm}^{-2}$ in the temperature range measured. The device was mounted in a dilution refrigerator with a

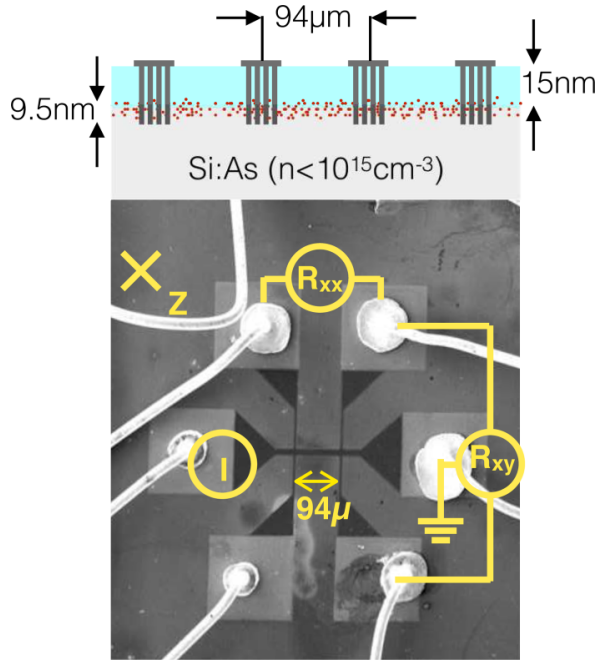

FIG. 1. The Hall bar and the principal directions, and a schematic of the device cross section showing the arsenic-doped handle (light grey), the deposited phosphorus $\delta$-layer (red), the overgrown silicon (cyan) and the aluminum contacts.

$3 \mathrm{D}$ vector magnet capable of a field magnitude of $2 \mathrm{~T}$ in all directions. The base temperature was $\sim 13 \mathrm{mK}$ ( $22 \mathrm{mK}$ with the field sweeping). Transverse and longitudinal resistances were measured for linear field sweeps, with the magnetic field out of plane $(Z)$, in plane ( $X$ and $Y$ ), and for angular sweeps at a field magnitude of $2 \mathrm{~T}$ in all three planes. The temperature was varied from $22 \mathrm{mK}$ to $30 \mathrm{~K}$. Additionally, angular sweeps were performed at $22 \mathrm{mK}$ with different field magnitudes, from $2 \mathrm{~T}$ to $0.1 \mathrm{~T}$. The current in all magnetoresistance (MR) measurements was $114 \mathrm{nA}$. The current modulation frequency in all measurements was $7.6 \mathrm{~Hz}$. The main axes of the magnetic field were finely adjusted by applying a maximum in-plane field in the $X$ and $Y$ directions and minimizing the Hall effect. An optical image and cross section of the sample appear in Fig. 1.

\section{RESULTS}

Figure 2 shows experimental magnetotransport (MT) results. Figure 2(a) shows MT curves with the field out of plane (transverse and longitudinal MR) and in plane (longitudinal MR). The fitted longitudinal MT is according to Hikami et al. [13] and Dugaev and Khemlnitskii [20]. The resulting layer thickness following Sullivan et al. [21] is $9.44 \pm 0.47 \mathrm{~nm}$, with a negligible temperature dependence. There was no measurable difference between the MR with the field in both in-plane directions. Figure 2(b) shows the temperature dependence of the transport parameters derived from the fits. We see that the conductivity scales as $\ln (T)$, as expected [22]. The phase coherence length $L_{\varphi}$ has a power-law temperature dependence, and much like previous results in different material systems and thicknesses, it saturates at low temperature [23-28]. The mean free path $L$ is only weakly temperature dependent, and as the temperature increases $L_{\varphi}$ tends towards $L$. We expect the WL approximation to break down as a result. The experimental values of $\tau_{B}$ are shown in Fig. 2(c) and we find that they are proportional to $B_{\|}^{2}$ as expected, with a weak temperature 


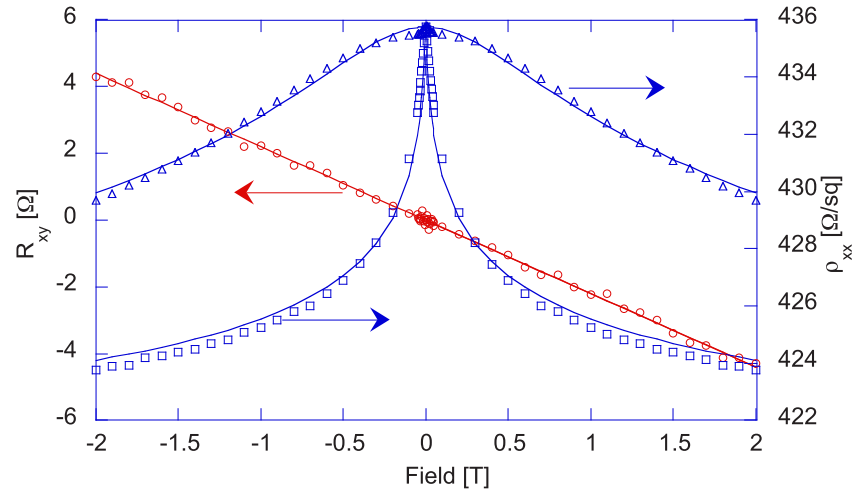

(a)

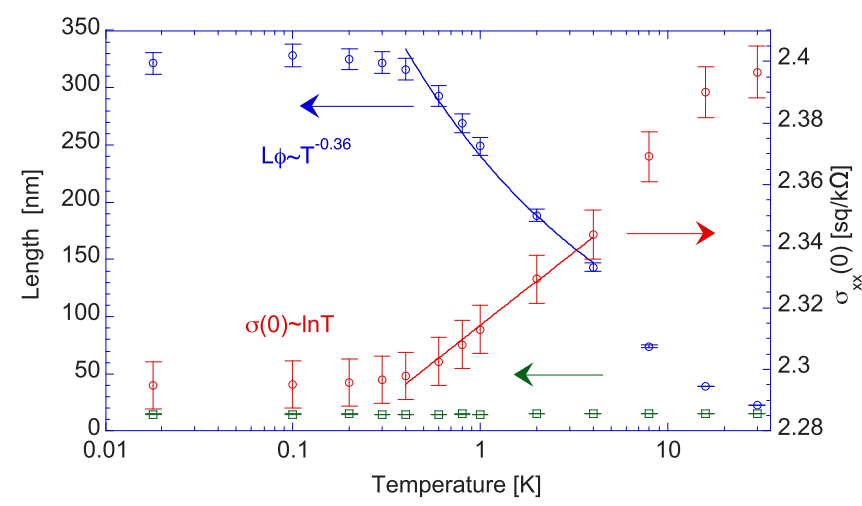

(b)

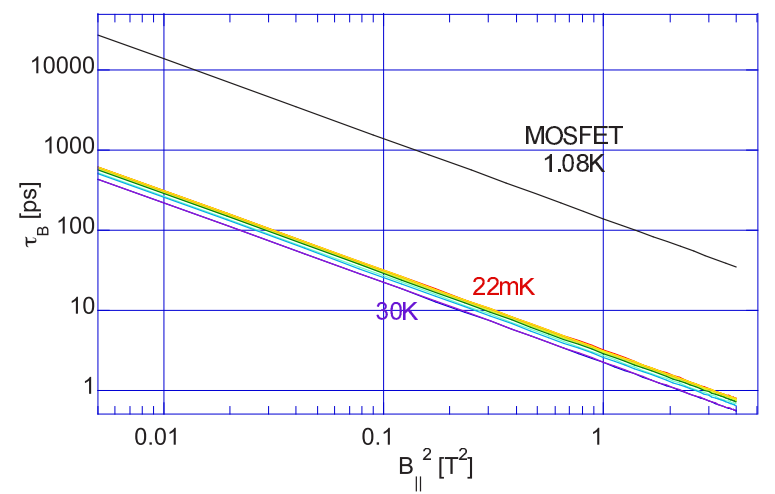

(c)

FIG. 2. MT results. (a) MT curves at $22 \mathrm{mK}$ and their fits. MR with magnetic field out-of-plane in blue squares, in plane in blue triangles, Hall resistance in red circles. (b) Temperature dependence of transport parameters. The blue circles are the phase coherence length. The unsaturated part which still has $L_{\varphi} \gg L$ shows a power-law dependence. The green squares are the mean free path, which is temperature-independent in this range. The red circles are conductivity at zero field, showing logarithmic WL behavior. The error bars are $95 \%$ confidence intervals. (c) A temperature dependence of the experimental phase-breaking time $\tau_{B}$ vs $B_{\|}^{2}$. The black line is MOSFET data from Mensz and Wheeler [14].

dependence [14]. The proportionality coefficient is 1.5 orders of magnitude lower than in previous MOSFET results, meaning that the in-plane field in our work has a larger phase-breaking effect. We attribute this to a rougher interface of the $\delta$-layer compared with the MOSFET [29], related to the absence of a locking layer. Indeed, the use of WL for evaluating surface roughness was disccussed by Anderson et al. [30].

Figure 3 shows the $p$-mean fits. In Fig. 3(a), we see $\Delta \sigma_{x x}$ (hereafter referred to as $\Delta \sigma$ ) at $22 \mathrm{mK}$ and $2 \mathrm{~T}$ as a function of the field angle in the $Z X$ plane (blue). The sum of $\Delta \sigma$ caused by a parallel field and a perpendicular field at the appropriate magnitudes is shown in green. The traces intersect at $0^{\circ}, 90^{\circ}, 180^{\circ}$, and $270^{\circ}$ (measured from the plane), since only one field component is nonzero. At other angles, this is clearly not the case. The red trace is a $p$-mean of the perpendicular and parallel field $\mathrm{MC}$, where the fitted value of $p$ is $4.18 \pm 0.10$. The $p$-mean fits at $2 \mathrm{~T}$ for a temperature range of $22 \mathrm{mK}-30 \mathrm{~K}$ are shown in Fig. 3(b) and the quality of the fits is maintained. $\Delta \sigma$ and $p$-mean fits at $22 \mathrm{mK}$, for different field magnitudes, are shown in Fig. 3(e). Figures 3(c) and 3(d) show that the normalized deviation between the tilted-field measurement and the $p$-mean fit remains within $5 \%$ at different angles and temperatures.

Figure 4 compares the $p$ values. The insets show them as a function of temperature and of field magnitude. In the main figure, we merge these data by plotting $p$ as a function of the number of magnetic flux quanta through the maximal area enclosed before dephasing, i.e., $A=L_{\varphi}^{2} / 4 \pi$.

\section{DISCUSSION}

To understand the origin of the $p$-mean we begin by considering the isotropic 3D case, where the MC depends only on the magnitude of $B$ [16,31-33]. At low and high fields, it can be approximated by a local power law:

$$
\Delta \sigma(\alpha B)=\alpha^{2 / p} \Delta \sigma(B),
$$

where $p=1,4$, respectively. For intermediate fields, the relation also holds for $\alpha \approx 1$ and a $p$ which depends on the field $B$, so that the degree of nonlinearity of the MC is encoded in $2 / p(B)$. Let $\theta$ be the angle between the field and an arbitrarily chosen plane in an isotropic sample, so $B_{\perp}=B \sin \theta$ and $B_{\|}=B \cos \theta$; then by using Eq. (3), the $p$-mean relation holds identically:

$$
\begin{aligned}
{\left[\Delta \sigma\left(B_{\perp}\right)\right]^{p}+\left[\Delta \sigma\left(B_{\|}\right)\right]^{p} } & =(\cos \theta)^{2}[\Delta \sigma(B)]^{p}+ \\
+(\sin \theta)^{2}[\Delta \sigma(B)]^{p} & =[\Delta \sigma(B)]^{p} .
\end{aligned}
$$

A corresponding theory for anisotropic 3D samples, where $\Delta \sigma$ depends also on the angle $\theta$ of the field $B$ with respect to a reference plane in the sample, was discussed by Mauz et al. [34]. A one-parameter scaling rule for the magnetic field was deduced, by which $\Delta \sigma$ for all angles collapses onto a single scaling curve:

$$
\Delta \sigma\left(B_{\perp}, B_{\|}\right)=f\left(B^{*}\right), \quad B^{*}=B \cdot \xi(\theta)^{-1},
$$

where the anisotropy enters only through the effective field $B^{*}$, related to the physical field $B$ by the angle-dependent scale factor $\xi(\theta) . \xi(\theta)$ determines the effectiveness of a field at angle $\theta$ in disrupting WL relative to that of a perpendicular field. Following Ref. [34], we define a scaling for our single quasi-2D layer as

$$
\frac{1}{\xi(\theta)}=\sqrt{\frac{\cos ^{2}(\theta)}{\xi_{0}^{2}}+\sin ^{2}(\theta)},
$$




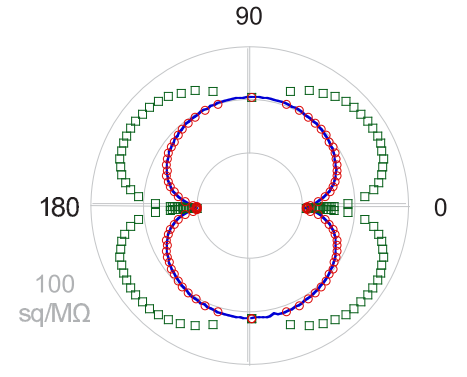

(a)

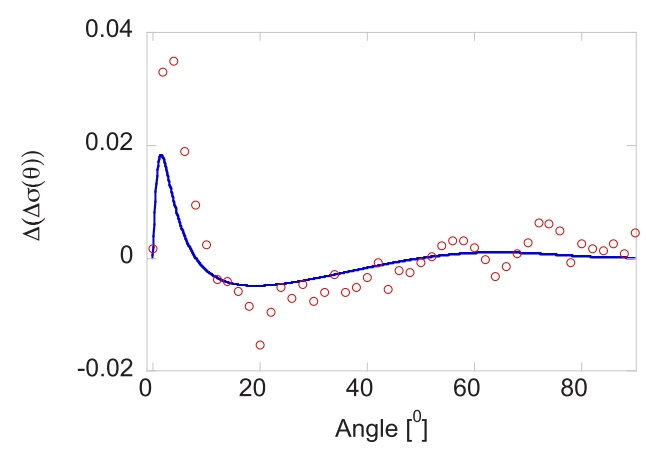

(c)

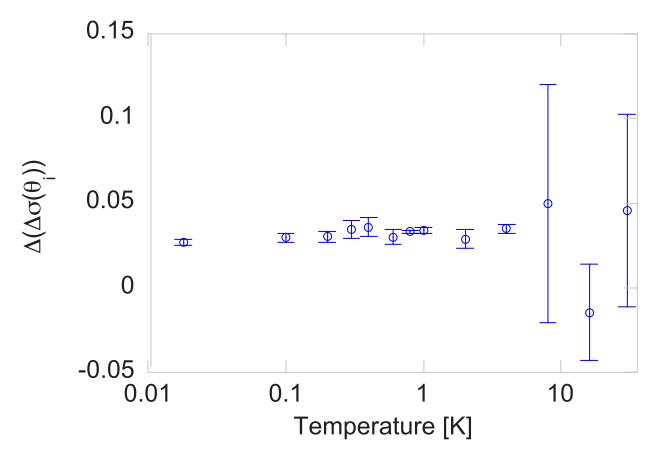

(d)

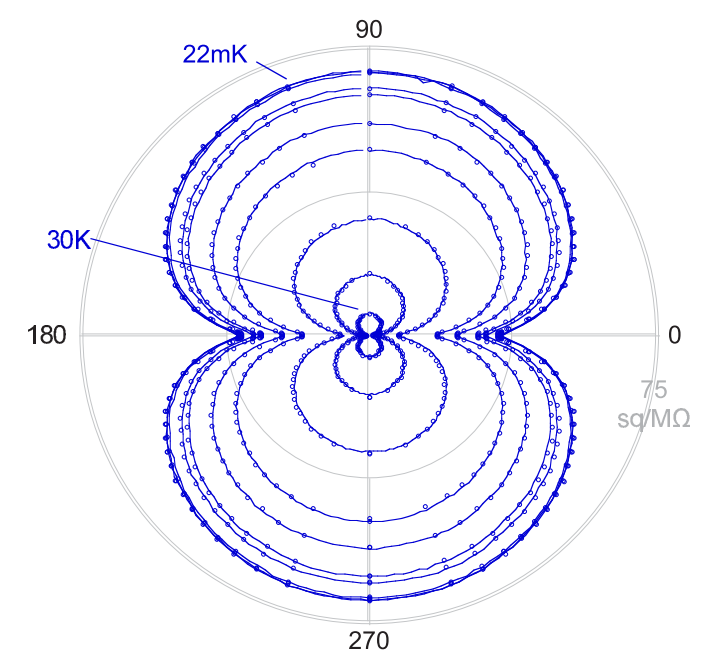

(b)

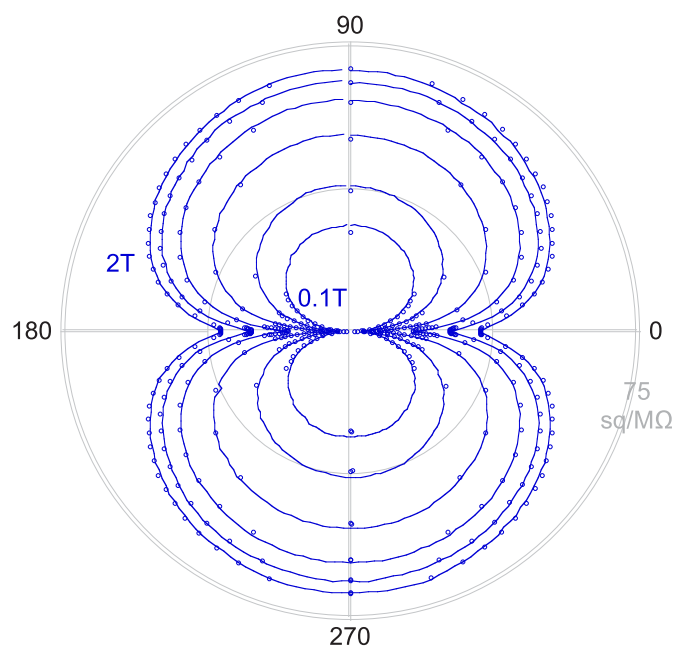

(e)

FIG. 3. $p$-mean fits: (a) Angular MC at 2 T, $22 \mathrm{mK}$. Blue line: measurement. Symbols: $p$-means of the perpendicular and parallel field $\Delta \sigma$; green squares: $p=1$ (algebraic sum); red circles: $p=4.18 \pm 0.10$. (b) Angular MC with $p$-mean fits at $2 \mathrm{~T}$ field magnitude, for $22 \mathrm{mK}$ (outermost), $200 \mathrm{mK}, 400 \mathrm{mK}, 800 \mathrm{mK}, 2 \mathrm{~K}, 4 \mathrm{~K}, 8 \mathrm{~K}, 16 \mathrm{~K}, 30 \mathrm{~K}$ (innermost). (c) Normalized deviation of the $p$-mean fit from the measured $\Delta \sigma$ [defined as $\Delta(\Delta \sigma(\theta)) \equiv \frac{\Delta \sigma(\theta)-\Delta \sigma p \text {-mean }}{\Delta \sigma(\theta)}$ ] vs field angle at $22 \mathrm{mK}$, showing that the most significant deviations are for small angles (i.e., nearly in-plane fields). The red symbols and the blue line are experimental data and calculation based on Ref. [14], respectively. (d) $\Delta\left(\Delta \sigma\left(\theta_{i}\right)\right)$ vs temperature. (e) Angular MC with $p$-mean fits at $22 \mathrm{mK}$, for $2 \mathrm{~T}$ (outermost), $1.5 \mathrm{~T}, 1.0 \mathrm{~T}, 0.5 \mathrm{~T}, 0.2 \mathrm{~T}, 0.1 \mathrm{~T}$ (innermost).

where $\xi_{0} \geqslant 1$ denotes the effective anisotropy ratio. We find a similar scaling collapse in our experimental data, as shown in Fig. 5: MC curves taken at a full range of angles $0^{\circ} \leqslant \theta \leqslant 90^{\circ}$ collapse onto the perpendicular field MC, which is well fitted by the expression derived in Ref. [13]. This is in contrast to Mauz et al. [34], where the anisotropic collapse is to the bulk expression derived by Kawabata. The temperature dependence of $\xi_{0}$ appears in the inset, showing that the system becomes more isotropic as the temperature increases. We have also found that, to a good approximation, this same scaling collapse is empirically obeyed in Mathur's theory for a single layer with finite layer roughness and finite temperature [15]. For large angles, we see

$$
\begin{aligned}
\Delta \sigma^{p} & =\left[f\left(\frac{B}{\xi(\theta)}\right)\right]^{p} \approx \frac{f(B)^{p}}{\xi(\theta)^{2}} \\
& =\left(\frac{\cos ^{2}(\theta)}{\xi_{0}^{2}}+\sin ^{2}(\theta)\right) f(B)^{p} .
\end{aligned}
$$

Assuming that $2 / p$ is the $\log$ derivative of $f$, then $\sin ^{2}(\theta) f(B)^{p} \approx f(B \sin (\theta))^{p}$. Additionally, if $f(B)$ were a 


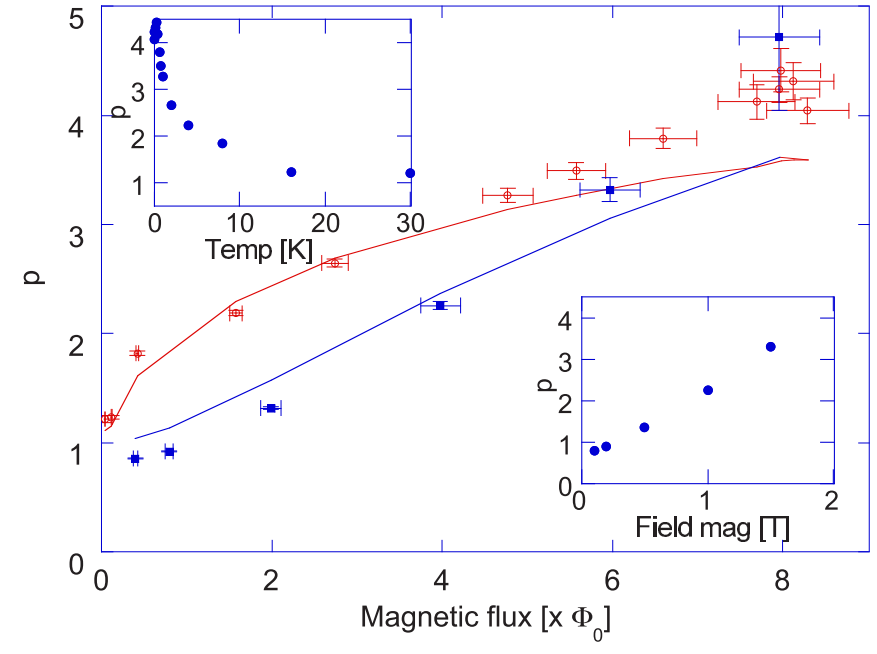

FIG. 4. $p$ vs magnetic flux quanta through a circular closed trajectory with circumference $L_{\varphi}$. Values from temperature and field dependences are in red (empty circles) and blue (full squares), respectively. The symbols are the fitted values of $p$ to measured results and the lines are calculated at $\theta_{i}$ [see Eq. (7)], with $\theta_{i}$ derived from the WL MC in Refs. [13] and [20]. The $X$-axis errors originate in the $L_{\varphi}$ errors in Fig. 2(b). Top and bottom insets are the temperature and field dependences vs temperature and field magnitudes, respectively.

power-law [Eq. (3)], then it would follow that

$$
\frac{\cos ^{2}(\theta)}{\xi_{0}^{2}} f(B)^{p} \approx f\left(B \frac{\cos (\theta)}{\xi_{0}}\right)^{p} .
$$

In that case, the $p$-mean relation [Eq. (2)] would hold as a result of scaling and power-law dependencies. Interestingly, we find that even though $f(B)$ does not follow a power-law dependence, the empirical value of $p$ is modified such that the $p$-mean rule still applies and we can regard $2 / p(B)$ as describing the interplay between $\Delta \sigma$ and the vector field $\boldsymbol{B}$ in the $2 \mathrm{D}$ case.

We see that, provided a local power law exists, the $p$-mean is intrinsic to the isotropic bulk case. Furthermore, in the $2 \mathrm{D}$ case, we successfully describe the tilted-field MC using the Hikami formula and a scaled field, and derive the $p$-mean from that scaling. We can now make several observations regarding the power law, $\xi_{0}$ and $p$. The ansatz in Eq. (3) helped to demonstrate the $p$-mean relation for strictly field-magnituderelated MC. We can show that for a particular field angle, this power-law relation emerges directly from our scaling and $p$-mean observations. We define a "crossing angle" $\theta_{i}$ where the influence of the parallel and perpendicular fields, applied separately, is equal: $\Delta \sigma_{\perp}\left(\theta_{i}\right)=\Delta \sigma_{\|}\left(\theta_{i}\right)$, where

$$
\Delta \sigma_{\perp}\left(\theta_{i}\right)=\Delta \sigma\left(B \sin \theta_{i}, 0\right), \text { and } \Delta \sigma_{\|}\left(\theta_{i}\right)=\Delta \sigma\left(0, B \cos \theta_{i}\right) .
$$

At this angle, the $p$-mean exponent can be conveniently expressed as

$$
p=\left[\log _{2}\left(\frac{\Delta \sigma\left(\theta_{i}\right)}{\Delta \sigma_{\perp}\left(\theta_{i}\right)}\right)\right]^{-1},
$$

provided Eq. (2) holds at the crossing point [which is empirically found to be the case, see Fig. 3(d)]. At the crossing angle

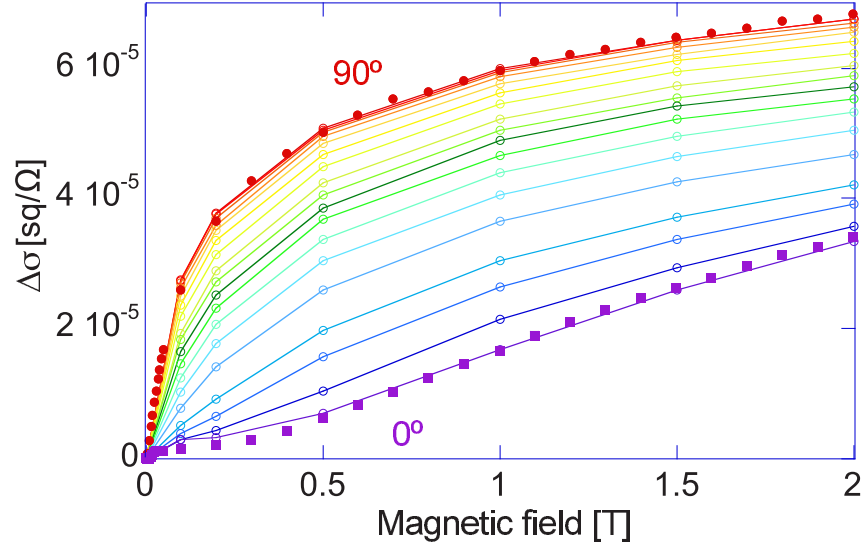

(a)

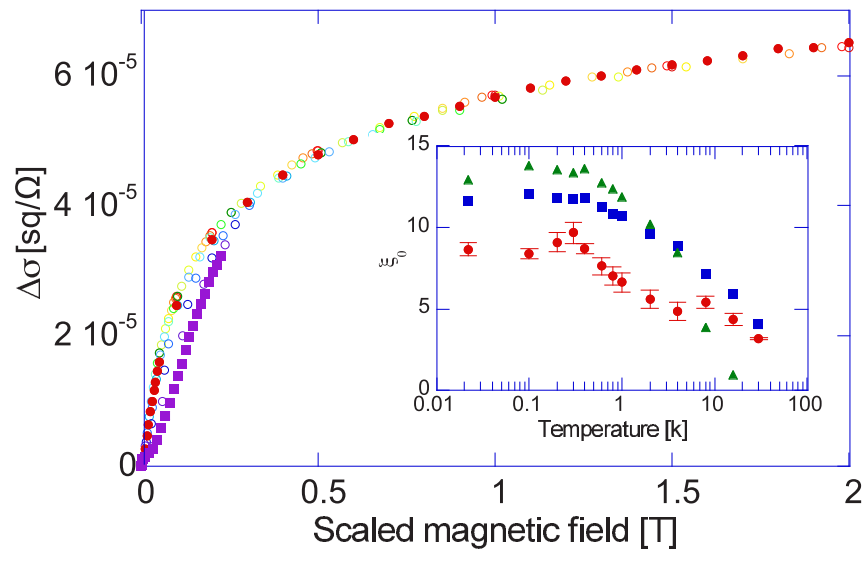

(b)

FIG. 5. Anisotropic field scaling: (a) shows $\Delta \sigma$ vs field magnitude at different field angles, $22 \mathrm{mK}$. (b) shows same data as (a) but with the magnetic field scaled according to Eq. (6) with $\xi_{0}=8.92$. The full red circles and violet squares are the $\Delta \sigma_{\perp}$ and $\Delta \sigma_{\|}$values from Fig. 2(a), respectively. Inset in (b): $\xi_{0}$ values at different temperatures, showing a stronger anisotropy at low temperatures. The red circles are best fits to the MC data, the blue squares and green triangles are from Eqs. (9) and (12), respectively.

we have, using Eq. (5),

$$
f\left(B \sin \theta_{i}\right)=\Delta \sigma_{\perp}\left(\theta_{i}\right)=\Delta \sigma_{\|}\left(\theta_{i}\right) \approx f\left(\frac{B \cos \theta_{i}}{\xi_{0}}\right),
$$

and hence

$$
\cot \theta_{i} \approx \xi_{0},
$$

i.e., the easily measurable $\theta_{i}$ directly quantifies the $\mathrm{MC}$ anisotropy.

Using Eq. (6), we see that $\xi\left(\theta_{i}\right)^{-1}=\sqrt{2} \sin \theta_{i}$. Once again using the empirical validity of Eq. (2) at the crossing point, we see that

$$
p=\left[\log _{2}\left(\frac{f\left(\frac{B}{\xi\left(\theta_{i}\right)}\right)}{f\left(B \sin \theta_{i}\right)}\right)\right]^{-1} .
$$

This expression for $p$ does not rely on the power-law assumption of Eq. (3). It then follows that

$$
f\left(\sqrt{2} B \sin \theta_{i}\right)=(\sqrt{2})^{2 / p} f\left(B \sin \theta_{i}\right),
$$


i.e., it emerges that $p$ encodes a power-law behavior of the MC scaling function at the crossing angle similar to Eq. (3).

$\xi_{0}$ can be related, at high fields, to $\tau_{\mathrm{el}}, \tau_{\varphi}$, and $\tau_{B}$ (the elastic, inelastic, and phase-breaking times, respectively) through an asymptotic approximation, i.e., at grazing angles (see Appendix for this derivation):

$$
\xi_{0} \approx \frac{4 \pi D}{\Phi_{0}} B \tau_{\mathrm{el}}\left(\frac{e^{\psi\left(\frac{1}{2}\right)}}{2+\frac{\tau_{\varphi}}{\tau_{B}}} \frac{\tau_{\varphi}}{\tau_{\mathrm{el}}}-\frac{1}{2}\right),
$$

where $D$ is the diffusion constant and $\psi$ is the digamma function. The values for Eq. 12 appear in the inset of Fig. 5(b) (green triangles) and we find the good agreement with the fitted data to be consistent with our understanding of $\xi_{0}$ : a large anisotropy results in a $\theta_{i}$ that is close to the plane, making the asymptotic approximation in our calculation valid.

Equations 9 and 10 give a full vector-characterization of anisotropic 2D-like WL. Given the results of a perpendicularand parallel-field MC measurement (e.g., the squares and triangles in Fig. 2(a), respectively), then the crossing angle $\theta_{i}$ can be found, for arbitrary magnetic fields, by interpolation. Both $\xi_{0}$ and $p(B)$ can then be calculated, and using Eqs. (5) and (6) or (2), respectively, a full angle and field-magnitude map of $\Delta \sigma$ is constructed.

The scaling parameter $\xi_{0}$ can also give an indication to the layer thickness; a natural interpretation of the scaling collapse in Eqs. 5 and 6 is that the MC is determined by the magnetic flux through an angle-dependent phase-breaking area. Taking the effective phase-breaking length perpendicular to the field as $L_{\text {eff }}(\theta)=L_{\varphi} /(\pi \xi(\theta))$, we might guess that the scaling approach would be valid until angles $\theta$ are reached such that the projection of $L_{\text {eff }}(\theta)$ becomes comparable to the layer thickness, $d \approx L_{\text {eff }} \cos \theta$. Thus the breakdown of scaling gives a second-independent-estimate of $d$, which yields $d=13.7 \mathrm{~nm}$ at $22 \mathrm{mK}$.

\section{CONCLUSION}

We have taken advantage of modern vector magnet and sample preparation technologies to revisit the problem of WL in silicon, where we have now implemented a 2D half-filled disordered Hubbard model with $\mathrm{t} / \mathrm{U}$ of order unity. The experiment examines the entire temperature and vector field dependence of the conductance for a $\mathrm{Si}: \mathrm{P}$ realization of such a model, and we find astonishingly precise agreement with 40-year-old theory, incorporating the effects of interface roughness but neglecting Coulomb interactions. The MC in a tilted field is found to be a $p$-mean of the $\mathrm{MCs}$ in the parallel and perpendicular field components, separately. In analogy to the dependence of $\Delta \sigma$ on the magnitude of $B$ in the three-dimensional case of Kawabata [16], we can regard $2 / p$ as a generalized measure of the sensitivity of $\Delta \sigma$ to the varying $\boldsymbol{B}$ vector in the $2 \mathrm{D}$ case. We also found a scaling collapse of the MC onto a single function of an angle-dependent effective magnetic field. Such a scaling form has previously been proposed for anisotropic 3D systems [34], but it is interesting that it holds also in the almost purely $2 \mathrm{D}$ system formed by our $\delta$-layer. Scaling is well obeyed for all field directions except when the field is very nearly in plane. At these angles, a crossover occurs to a different regime dominated by the parallel field; the out-of-plane confinement dominates the response to the field and the anisotropic 3D assumption breaks down.

\section{ACKNOWLEDGMENT}

This work was funded by EPSRC grant EP/M009564/1 (ADDRFSS). The data underlying this work is available without restriction (doi:10.5281/zenodo.1220436).

\section{APPENDIX: DEPENDENCE OF THE SCALING PARAMETER $\xi_{0}$ ON $\tau_{\varphi}, \tau_{e}$}

Relying on the empirical validity of the scaling ansatz for a range of fields and angles, we can extract a dependence of the scaling parameter $\xi_{0}$ on the system parameters. The scaling ansatz enables us to obtain an expression for the vector MC $\Delta \sigma$ in terms of the perperndicular MC $\Delta \sigma_{\perp}^{(s)}$ [13] and the scaling function $\xi(\theta)$ :

$$
\begin{aligned}
\Delta \sigma & \sim \psi\left(\frac{1}{2}+\frac{B_{e}}{B_{\perp}}\right)-\psi\left(\frac{1}{2}+\frac{B_{\varphi}}{B_{\perp}}+\beta \frac{B^{2} \cos ^{2} \theta}{B_{\perp}}\right), \\
\Delta \sigma_{\perp}^{(s)} & \sim \psi\left(\frac{1}{2}+\frac{B_{e}}{B^{*}}\right)-\psi\left(\frac{1}{2}+\frac{B_{\varphi}}{B^{*}}\right),
\end{aligned}
$$

where $\beta$ represents the dephasing strength of the parallel magnetic field, as has been discussed in several microscopic derivations $[14,15,20]$, and $B^{*}=B / \xi(\theta)$ is the rescaled effective magnetic field. We can now try to compare the MCs assuming that $\theta \approx \theta_{i}$, an assumption which yielded a good description for $\xi_{0}$ as described in the main text with Eq. (9). For low temperatures, we find that the crossing angle is relatively small. Equating $\Delta \sigma$ and $\Delta \sigma_{\perp}^{(s)}$ and numerically extracting the dependence of $\xi_{0}$ on temperature even for very small angles $\theta \approx 0.01$ yields a good approximation to the dependences depicted in the inset of Fig. 5(b).

To obtain insight into the dependence of $\xi_{0}$ on the physical parameters, we proceed with approximating each of the digamma functions, which appear above in the expressions for $\Delta \sigma$ and $\Delta \sigma_{\perp}^{(s)}$. For small angles and the values of $B, B_{\phi}, B_{e}$ relevant for Fig. 5(b), $\psi\left(\frac{1}{2}+\frac{B_{\varphi}}{B^{*}}\right)$ can be expanded close to $\frac{1}{2}$, i.e., where

$$
\psi\left(\frac{1}{2}+\epsilon\right) \approx \psi\left(\frac{1}{2}\right)+\frac{\pi^{2}}{2} \epsilon,
$$

whereas the other digamma functions are approximated asymptotically as logarithms since they possess large numerical arguments:

$$
\begin{aligned}
\Delta \sigma & \sim \ln \left(\frac{B_{e}}{B_{\perp}}\right)-\ln \left(\frac{B_{\varphi}}{B_{\perp}}+\beta \frac{B^{2} \cos ^{2} \theta}{B_{\perp}}\right), \\
\Delta \sigma_{\perp}^{(s)} & \sim \ln \left(\frac{1}{2}+\frac{B_{e}}{B} \xi_{0}\right)-\psi\left(\frac{1}{2}\right),
\end{aligned}
$$

where we find that it is sufficient to take the zeroth-order approximation in the Taylor expansion for the last term. By equating $\Delta \sigma$ and $\Delta \sigma_{\perp}^{(s)}$ and extracting $\xi_{0}$, we immediately obtain

$$
\xi_{0} \approx \frac{B}{B_{e}}\left(-\frac{1}{2}+\frac{B_{e} e^{\psi\left(\frac{1}{2}\right)}}{B_{\varphi}+\beta B^{2}}\right),
$$


which can also be expressed in terms of the microscopic relaxation times as

$$
\xi_{0} \approx \frac{4 \pi D}{\Phi_{0}} B \tau_{\mathrm{el}}\left(\frac{e^{\psi\left(\frac{1}{2}\right)}}{2+\frac{\tau_{\varphi}}{\tau_{B}}} \frac{\tau_{\varphi}}{\tau_{\mathrm{el}}}-\frac{1}{2}\right) .
$$

This expression reveals the dependence of the scaling parameter $\xi_{0}$ on the microscopic parameters and strengthens our assertion that the anisotropy of the system is highest at low temperature. This dependence is plotted in Fig. 5(b), and while it works well to qualitatively describe the dependence for a large range of temperatures, it is understandably less accurate for high temperature where the crossing angles are large.
We note that according to this derivation, $\xi_{0}$ also observes a dependence on the magnetic field. We argue that this manifests the approximate nature of the scaling ansatz where $\xi_{0}$ is taken as constant. The constant $\xi_{0}$ is determined by a fit and is mostly influenced by data at medium field strengths. The influence of $\xi_{0}$ is most important at medium range fields because at small fields the MC is small and at large fields the MC varies slowly with fields. In practice, this typical scaling factor, when applied to all measured angles and fields, performs well. We also see that the dependence of $\xi_{0}$ with temperature, i.e., $\boldsymbol{\tau}_{\boldsymbol{\varphi}}$ is consistent with trends found here.
[1] S. R. Schofield, N. J. Curson, M. Y. Simmons, F. J. Rueß, T. Hallam, L. Oberbeck, and R. G. Clark, Atomically Precise Placement of Single Dopants in Si, Phys. Rev. Lett. 91, 136104 (2003).

[2] G. Scappucci, W. M. Klesse, L. R. A. Yeoh, D. J. Carter, O. Warschkow, N. A. Marks, D. L. Jaeger, G. Capellini, M. Y. Simmons, and A. R. Hamilton, Bottom-up assembly of metallic germanium, Sci. Rep. 5, 12948 (2015).

[3] H.-D. Chiou, Phosphorus concentration limitation in Czochralski silicon crystals, J. Electrochem. Soc. 147, 345 (2000).

[4] S. R. McKibbin, C. M. Polley, G. Scappucci, J. G. Keizer, and M. Y. Simmons, Low resistivity, super-saturation phosphorusin-silicon monolayer doping, Appl. Phys. Lett. 104, 123502 (2014).

[5] S. Solmi, A. Parisini, R. Angelucci, A. Armigliato, D. Nobili, and L. Moro, Dopant and carrier concentration in $\mathrm{Si}$ in equilibrium with monoclinic SiP precipitates, Phys. Rev. B 53, 7836 (1996).

[6] T. Ando, A. B. Fowler, and F. Stern, Electronic properties of two-dimensional systems, Rev. Mod. Phys. 54, 437 (1982).

[7] R. G. Wheeler, Magnetoconductance and weak localization in silicon inversion layers, Phys. Rev. B 24, 4645 (1981).

[8] M. Y. Simmons, A. R. Hamilton, M. Pepper, E. H. Linfield, P. D. Rose, and D. A. Ritchie, Metallic behaviour and localisation in 2D GaAs hole systems, Physica E (Amsterdam) 11, 161 (2001), Proceedings of the Rutherford Memorial Workshop on Semiconductor Nanostructures, Queenstown, New Zealand, 5-9 February (2001).

[9] S. Lara-Avila, A. Tzalenchuk, S. Kubatkin, R. Yakimova, T. J. B. M. Janssen, K. Cedergren, T. Bergsten, and V. Fal'ko, Disordered Fermi liquid in epitaxial graphene from quantum transport measurements, Phys. Rev. Lett. 107, 166602 (2011).

[10] A. L. Saraiva, A. Baena, M. J. Calderón, and B. Koiller, Theory of one and two donors in silicon, J. Phys.: Condens. Matter 27, 154208 (2015).

[11] N. H. Le, A. J. Fisher, and E. Ginossar, Extended Hubbard model for mesoscopic transport in donor arrays in silicon, Phys. Rev. B 96, 245406 (2017).

[12] A. Georges, G. Kotliar, W. Krauth, and M. J. Rozenberg, Dynamical mean-field theory of strongly correlated fermion systems and the limit of infinite dimensions, Rev. Mod. Phys. 68, 13 (1996).

[13] S. Hikami, A. I. Larkin, and Y. Nagaoka, Spin-orbit interaction and magnetoresistance in the two dimensional random system, Prog. Theor. Phys. 63, 707 (1980).
[14] P. M. Mensz and R. G. Wheeler, Magnetoconductance due to parallel magnetic fields in silicon inversion layers, Phys. Rev. B 35, 2844 (1987).

[15] H. Mathur and H. U. Baranger, Random Berry phase magnetoresistance as a probe of interface roughness in Si MOSFETs, Phys. Rev. B 64, 235325 (2001).

[16] A. Kawabata, Theory of negative magnetoresistance in three-dimensional systems, Solid State Commun. 34, 431 (1980).

[17] J. A. Hagmann, X. Wang, P. Namboodiri, J. Wyrick, R. Murray, M. D. Stewart, Jr., R. M. Silver, and C. A. Richter, High resolution thickness measurements of ultrathin Si:P monolayers using weak localization, Appl. Phys. Lett. 112, 043102 (2018).

[18] J. G. Keizer, S. Koelling, P. M. Koenraad, and M. Y. Simmons, Suppressing segregation in highly phosphorus doped silicon monolayers, ACS Nano. 9, 12537 (2015).

[19] K. E. J. Goh, L. Oberbeck, M. Y. Simmons, A. R. Hamilton, and M. J. Butcher, Influence of doping density on electronic transport in degenerate Si:P $\delta$-doped layers, Phys. Rev. B 73, 035401 (2006).

[20] V. K. Dugaev and D. E. Khemlnitskii, Magnetoresistance of metal films with low impurity concentrations in a parallel magnetic field, Sov. Phys. J. Exp. Theor. Phys. 59, 1038 (1984).

[21] D. F. Sullivan, B. E. Kane, and P. E. Thompson, Weak localization thickness measurements of Si:P delta-layers, Appl. Phys. Lett. 85, 6362 (2004).

[22] B. L. Altshuler, D. Khmel'nitzkii, A. I. Larkin, and P. A. Lee, Magnetoresistance and Hall effect in a disordered twodimensional electron gas, Phys. Rev. B 22, 5142 (1980).

[23] C. M. Polley, W. R. Clarke, and M. Y. Simmons, Comparison of nickel silicide and aluminium ohmic contact metallizations for low-temperature quantum transport measurements, Nanoscale Res. Lett. 6, 538 (2011).

[24] R. Cheung, L. J. Geerligs, J. Caro, A. H. Verbruggen, K. Werner, and S. Radelaar, Weak localisation and correlation effects in a two dimensional hole gas in $\mathrm{Si} / \mathrm{Si}_{1-x} \mathrm{Ge}_{x}$ heterostructures, Physica B (Amsterdam) 194-196, 1225 (1994).

[25] P. Fournier, C. J. Lobb, and R. L. Greene, Saturation of the phase coherence length at low temperatures in $\operatorname{Pr}_{1.95} \mathrm{Ce}_{0.05} \mathrm{CuO}_{4}$, Physica C (Amsterdam) 341-348, 1941 (2000).

[26] T. Ihn, D. Graf, F. Molitor, C. Stampfer, and K. Ensslin, Phasecoherent transport in a mesoscopic few-layer graphite wire, 
Physica E (Amsterdam) 40, 1851 (2008); 13th International Conference on Modulated Semiconductor Structures.

[27] D.-K. Ki, D. Jeong, J.-H. Choi, H.-J. Lee, and K.-S. Park, Inelastic scattering in a monolayer graphene sheet: A weak-localization study, Phys. Rev. B 78, 125409 (2008).

[28] J. J. Lin, Y. L. Zhong, and T. J. Li, Effect of annealing on electron dephasing in three-dimensional polycrystalline metals, Europhys. Lett. 57, 872 (2002).

[29] P. M. Mensz, R. G. Wheeler, C. T. Foxon, and J. J. Harris, Determination of spatial potential fluctuations in $\mathrm{Si}$ and $\mathrm{GaAs}$ inversion layers by weak localization, Appl. Phys. Lett. 50, 603 (1987).

[30] W. R. Anderson, D. R. Lombardi, R. G. Wheeler, T. P. Ma, and P. H. Mitev, Determination of $\mathrm{Si} / \mathrm{SiO} 2$ interface roughness using weak localization, in International Symposium on VLSI
Technology, Systems, and Applications, 1993, Proceedings of Technical Papers 12-14 May 1993, Taipei, Taiwan (IEEE, Piscataway, NJ, 1993), pp. 82-85.

[31] A. Kawabata, Theory of negative magnetoresistance I. Application to heavily doped semiconductors, J. Phys. Soc. Jpn. 49, 628 (1980).

[32] C. Yamanouchi, K. Mizuguchi, and W. Sasaki, Electric conduction in phosphorus doped silicon at low temperatures, J. Phys. Soc. Jpn. 22, 859 (1967).

[33] T. F. Rosenbaum, R. F. Milligan, G. A. Thomas, P. A. Lee, T. V. Ramakrishnan, R. N. Bhatt, K. DeConde, H. Hess, and T. Perry, Low-temperature magnetoresistance of a disordered metal, Phys. Rev. Lett. 47, 1758 (1981).

[34] C. Mauz, A. Rosch, and P. Wölfle, Dimensional crossover of weak localization in a magnetic field, Phys. Rev. B 56, 10953 (1997). 